\title{
Vortices in the Landau-Ginzburg Model of the Quantized Hall Effect
}

\author{
M. HASSAÏNE $\left({ }^{1}\right)$, P. A. HORVÁTHY $\left({ }^{2}\right)$ and J.-C. YERA $\left({ }^{3}\right)$ \\ Département de Mathématiques \\ Université de Tours
}

Parc de Grandmont, F-37200 TOURS (France)

\begin{abstract}
The 'Landau-Ginzburg' theory of Girvin and MacDonald, modified by adding the natural magnetic term, is shown to admit stable topological as well as non-topological vortex solutions. The system is the commun $\lambda \rightarrow 0$ limit of two slightly different non-relativistic Maxwell-Chern-Simons models of the type introduced recently by Manton. The equivalence with the model of Zhang, Hansson and Kivelson is demonstrated.
\end{abstract}

Journal of Physics A (Math. Gen.) 31, 9073-79 (1998)

In Ref. [GIR], Girvin and MacDonald presented a "Landau-Ginzburg" theory for the Quantum Hall Effect. On phenomenological grounds, they suggest to represent the off-diagonal long range order (ODLRO) by a scalar field $\psi(\vec{x})$ on the plane, and the frustration due to deviations away from the quantized Laughlin density by an effective gauge potential $\vec{a}(\vec{x})$. We propose to describe this static planar system by the Lagrange density

$$
\mathcal{L}=\frac{1}{2} b^{2}+|\vec{D} \psi|^{2}+i \phi\left(|\psi|^{2}-1\right)-i \frac{\kappa}{2}(\phi \vec{\nabla} \times \vec{a}+\vec{a} \times \vec{\nabla} \phi)
$$

where $b=\vec{\nabla} \times \vec{a}$ is the effective magnetic field, $\vec{D}=\vec{\nabla}+i \vec{a}$ is the gauge-covariant derivative, and the Lagrange multiplier $\phi$ is a scalar potential. Eq. (1) only differs from the original expression of Girvin and MacDonald in our having added the natural magnetic term, $b^{2} / 2$, also present in conventional Landau-Ginzburg theory $[\mathrm{LP}]$. The associated equations of motion read

$$
\begin{gathered}
\vec{D}^{2} \psi=i \phi \psi, \\
\kappa b=|\psi|^{2}-1, \\
\vec{\nabla} \times b-i \kappa \vec{\nabla} \times \phi=-\vec{\jmath},
\end{gathered}
$$

$\left({ }^{1}\right)$ e-mail: hassaine@cecs.cl

$\left({ }^{2}\right)$ e-mail: horvathy@univ-tours.fr

$\left.{ }^{3}\right)$ e-mail: yera@univ-tours.fr 
where $\vec{\jmath}=-i\left(\psi^{*} \vec{D} \psi-\psi(\vec{D} \psi)^{*}\right)$ the current. The first is a static, gauged Schrödinger equation. The second is the relation proposed by Girvin and MacDonald to relate the magnetic field to the particle density. Note here the -1 coming from the weird term $-i \phi$ in the Lagrangian, and representing the background charge [MAN]. The last equation is the Ampère-Hall law : $\vec{e}=-i \vec{\nabla} \phi$ is an effective electric field, so that $\kappa$ is interpreted as the Hall conductance.

This system is rather similar to those studied in Chern-Simons field theory [JP], and in particular to that recently introduced by Manton [MAN], [HHY]. Using these techniques, (i) we construct, with the method of Bogomolny [BOG], stable vortex solutions; (ii) point out that (1) is the $\lambda \rightarrow 0$ limit of two slightly different systems; (iii) demonstrate the equivalence with another 'Landau-Ginzburg' model, introduced by Zhang et al. $[\mathrm{ZH}]$.

Let us try in fact to reduce the second-order equations to the first-order "self-dual" system

$$
\left(D_{1} \pm i D_{2}\right) \psi=0, \quad \kappa b=|\psi|^{2}-1 .
$$

From the first of these relations we infer that $\vec{D}^{2}= \pm b$ and $\vec{\jmath}=\mp \vec{\nabla} \times \varrho$. Inserting into the Schrödinger equation determines the multiplier field as $\phi=\mp \frac{i}{\kappa}(\varrho-1)$. Then, from Ampère's law we get that the Hall conductance $\kappa$ has to be

$$
\kappa= \pm \frac{1}{2} .
$$

The vector potential is expressed using the self-dual Ansatz (3) as $\vec{a}=\mp(1 / 2) \vec{\nabla} \times \log \varrho+\vec{\nabla} \omega$, where $\omega$ is an arbitrary real function chosen so that $\vec{a}$ is regular [JP]. Inserting this into (2b) we end up, for both signs, with the "Liouville-type" equation

$$
\Delta \log \varrho=4(\rho-1)
$$

Note that any solution will carry an effective magnetic as well as an electric field.

Now we have to tell what kind of solutions we are interested in. To see this, let us consider the energy,

$$
H=\int\left\{\frac{b^{2}}{2}+|\vec{D} \psi|^{2}\right\} d^{2} \vec{x} .
$$

Using the identity $|\vec{D} \psi|^{2}=\left|\left(D_{1} \pm i D_{2}\right) \psi\right|^{2} \mp b|\psi|^{2}+$ (surface term), as well as (2b), the energy is rewritten as

$$
H=\int\left\{\left|\left(D_{1} \pm i D_{2}\right) \psi\right|^{2}+\left(\frac{1}{2 \kappa^{2}} \mp \frac{1}{\kappa}\right)|\psi|^{4}+\frac{1}{2 \kappa^{2}}+\left(-\frac{1}{\kappa^{2}} \pm \frac{1}{\kappa}\right)|\psi|^{2}\right\} d^{2} \vec{x} .
$$

The quartic term disappears when $\kappa= \pm 1 / 2$, leaving us with

$$
H=\int\left\{\left|\left(D_{1} \pm i D_{2}\right) \psi\right|^{2}+2\left(1-|\psi|^{2}\right)\right\} d^{2} \vec{x} .
$$

The system admits the zero-energy ground state (condensate) $\psi \equiv 1$ and $\vec{e}=0, b=0$.

Then we have two possibilities:

- Either, to get finite-energy, we can require that at infinity the solution tends to the condensate state, $|\psi| \rightarrow 1$ and $|\vec{D} \psi| \rightarrow 0$. These two conditions imply that the magnetic flux is quantized,

$$
\Phi \equiv \int b d^{2} x=2 \pi n
$$


where the integer $n$ is the 'winding number' of $\psi$, which maps the circle at infinity into $U(1)$. Then, using Eq. (2b), the 'particle number'

$$
N=\int\left(1-|\psi|^{2}\right) d^{2} \vec{x}
$$

is finite, and is related to the flux as $N=-\kappa \Phi=-2 \pi \kappa n$. ( $N$ is conserved owing to the continuity equation which follows from Eq. (2a).) Our finite-energy solutions (referred to as topological vortices) carry hence a non-vanishing flux as well as a charge. Their energy satisfies, by Eq. (7), the 'Bogomolny' inequality

$$
H \geq 2 N=-4 \pi \kappa n=\mp 2 \pi n .
$$

To get a positive bound, $\kappa$ and $n$ must have opposite signs : the upper (lower) sign works for $n<0$ (resp. for $n>0)$.

The 'Bogomolny' bound (10) is saturated when the self-duality eqns. (3) hold. (This is in fact the case of 'Bogomolny' vortices in the Abelian Higgs model, so that Eq. (5) admits a $2 n$-parameter family of solutions [WEIN]). Since they correspond to the absolute minima of the energy, such solutions are stable.

- Another possibility is, however, to choose $b=b_{0}=-1 / \kappa, \vec{e}=0, \psi \equiv 0$ as the ground state. This is not a finite-energy solution, though subtracting its (constant) energy density $1 / 2 \kappa^{2}=2$ from (7), we get

$$
\bar{H}=\int\left\{\frac{b^{2}}{2}-\frac{b_{0}^{2}}{2}+|\vec{D} \psi|^{2}\right\} d^{2} x=\int\left\{\left|\left(D_{1} \pm i D_{2}\right) \psi\right|^{2}-2|\psi|^{2}\right\} d^{2} x .
$$

Now we can look for non-topological solutions, i.e. such whose 'number', defined as

$$
\bar{N}=\int|\psi|^{2} d^{2} x
$$

converges. This 'renormalized number' $\bar{N}$ is now a continuous, rather than quantized parameter, which takes any positive value. Then we get the modified Bogomolny bound

$$
\bar{H} \geq-2 \bar{N},
$$

with the equality attained when the self-duality eqns. (3) hold. (Remember that $\bar{H}$ is the relative energy with respect to the infinite-energy background).

Since $\psi \rightarrow 0$ at infinity, the condition $D \psi \rightarrow 0$ does not now imply a quantized flux. The integral (8) is indeed infinite, as one sees directly in the radial case from Eqn. (12) below. However, subtracting the constant background magnetic field, $b=b_{0}=-1 / \kappa$, we get the renormalized number $\bar{N}$ in Eqn. (9'):

$$
\bar{\Phi} \equiv \int \bar{b} d^{2} x \equiv \int\left(b-b_{0}\right) d^{2} x=\int \frac{1}{\kappa}|\psi|^{2} d^{2} x=\frac{1}{\kappa} \bar{N} .
$$

This "renormalized flux" depends hence on a continuous parameter, namely on $\bar{N}$, just like for relativistic non-topological solitons [JLW].

The most convenient way of studying the solutions is to work directly with the first-order equations (3) rather then with the second-order Eqn. (5). Assuming that the fields have the form $\psi=f(r) e^{i n \theta}, a_{r}=$ $0, a_{\theta}=a(r)$, the $\mathrm{SD}$ equations read

$$
f^{\prime}= \pm \frac{n+a}{r} f, \quad \frac{a^{\prime}}{r}= \pm 2\left(f^{2}-1\right) .
$$


Regularity at the origin requires $n$ and $\kappa$ to be correlated as $\operatorname{sign} n=-\operatorname{sign} \kappa$, so that we get chiral solitons. The small- $r$ behavior is $f(r)=\alpha r^{|n|}, a=\mp r^{2}$, where $\alpha$ is a real parameter. For large $r$, we find instead

$$
\begin{aligned}
f(r) & \sim 1-C K_{0}(2 r) \sim 1-C \frac{e^{-2 r}}{\sqrt{r}} \\
b & \sim D r K_{1}(2 r) \sim D \frac{e^{-2 r}}{\sqrt{r}} \\
f(r) & \sim e^{-r^{2}} \\
b & \sim \mp 2\left(1-e^{-2 r^{2}}\right)
\end{aligned}
$$

for a topological vortex

for a non-topological vortex

A simple numerical calculation shows that, for each integer value of $n$, there is just one radially symmetric topological vortex obtained for $\alpha=\alpha_{0}(n)$, while non-topological vortices arise for an entire range $\alpha<\alpha_{0}(n)$ of the parameter. This behaviour is understood by looking, following Ezawa et al. [EZA], at the second-order equation (5). Again restricting ourselves to the radial case, we can view $x=\log f$ as the "position" and the radial coordinate, $t \equiv r>0$, as "time", so that Eq. (5) becomes

$$
\ddot{x}=-\frac{1}{t} \dot{x}-\vec{\nabla} U, \quad U(x) \equiv\left(2 x-e^{2 x}\right) .
$$

This is the equation of motion of a classical particle in a time-dependent frictional force and an external potential $U(x)$. Observe that $U(x)$ increases from $x=-\infty$, reaches its maximum at $x=0$, after which it decreases. As $t \rightarrow \infty, f \rightarrow 1$ i.e. $x \rightarrow 0$ for a topological soliton, and $f \rightarrow 0$ i.e. $x \rightarrow-\infty$ for a non-topological soliton. The regularity of $\psi$ requires $f$ to vanish at the origin. Let us hence consider a "particle" which starts at 'time' $t=0$ from the 'position' $x=-\infty$. If its "energy" is not sufficient to climb the potential hill, it will, after some time, fall back to $x=-\infty$ as $t \rightarrow \infty$ : we get a non-topological soliton. Increasing the initial velocity, we can make our particle to approach $x=0$ when $t \rightarrow \infty$, yielding a topological soliton. Clearly, this only happens for some specific initial "energy", corresponding to a specitif value $\alpha=\alpha_{0}(n)$ of the initial parameter. If the "energy" is even higher, the particle overshoots: the required boundary conditions can not be satisfied, so that no self-dual vortex can exist.

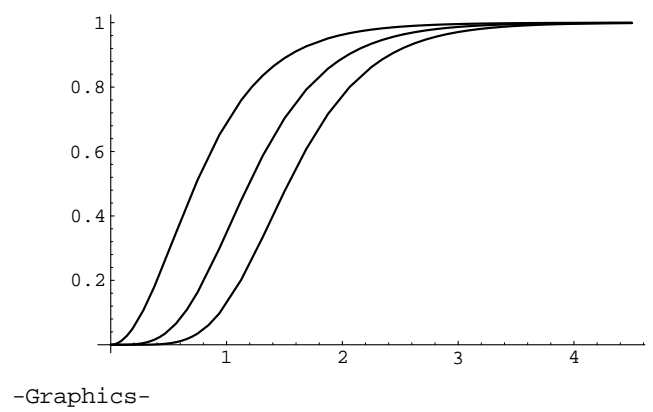

Fig. 1. The order parameter density of the radially symmetric topological vortices with winding number $n=1,2,3$. For each value of $n$, there is exactly one vortex. 


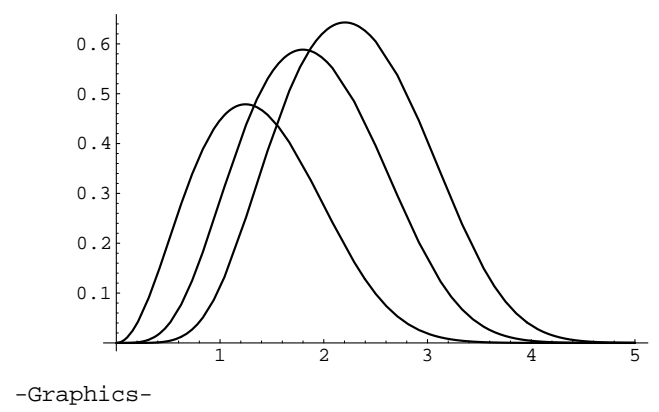

Fig. 2. The order parameter density of the radially symmetric non-topological vortices for $n=1,2,3$. For each value of $n$, there is a full range of vortices.

The arisal of these two different types of vortex solutions can be understood by adding a self-interaction potential, $U(\psi)$, to the Lagrangian. The use of such a potential is quite commun in Landau-Ginzburg theory, see Ref. [LP], p. 179. In the context of the Quantum Hall Effect, it can be viewed as the remnant of the twobody potential in the second-quantized Hamiltonian for spin-polarized electrons, when the effective theory is derived $[\mathrm{ZH}]$.

- For the symmetry breaking potential

$$
U(\psi)=\frac{\lambda}{8}\left(1-|\psi|^{2}\right)^{2}
$$

finite energy requires $|\psi| \rightarrow 1$ at infinity. Then the Bogomolny Eq. (3) yields topological vortex solutions, provided

$$
\lambda=-\frac{4}{\kappa^{2}} \pm \frac{8}{\kappa} .
$$

The potential in (14) is physically admissible (repulsive) when $\lambda \geq 0$, i.e. $|\kappa| \geq 1 / 2$.

- For the non-symmetry-breaking potential

$$
\bar{U}=C+\frac{\lambda}{8}|\psi|^{4}
$$

finite-energy requires instead $|\psi| \rightarrow 0$ at infinity, and the Bogomolny trick works when $C=-1 / 2 \kappa^{2}$ and for $\lambda$ as in Eq. (15). The potential (16) is physically admissible (attractive) when $\lambda \leq 0$, i.e., when $|\kappa| \leq 1 / 2$. Changing $\lambda$ from a negative to a positive value can be viewed as a phase transition, with transition point $\lambda=0$.

Let us stress that there is no way to obtain the quantization of the Hall conductance from our classical field theory : the condition (4) is merely replaced by Eqn. (15).

Thus, the modified Girvin model (1) can be viewed as the $\lambda \rightarrow 0$ limit of two, slightly different systems, one of them correct for $\kappa \geq 1 / 2$, the other for $\kappa \leq 1 / 2$. In both cases, there is a natural boundary condition 
at infinity, dictated by finite-energy. However, $|\kappa| \rightarrow 1 / 2$ when $\lambda \rightarrow 0$, so that both conditions can be used. In this limit, the boundary condition at infinity has to be put in by hand, since one can not know what stays 'behind' the coefficient $\lambda=0$. (This happens also for 't Hooft-Polyakov monopoles in the Prasad-Sommerfield limit).

In Ref. [HHY], we have shown that, for the potential (14) with $\lambda \neq 0$, the system admits a 6 -parameter group of symmetry, made of the unbroken parts of the "geometric" and "hidden" Schrödiger symmetries. It is easy to see that, for $\lambda=0$ some of the obstructions are lifted so that "imported" dilatations and expansions are also unbroken. Thus, we have the full "hidden" Schrödiger symmetry, just like for the purely quartic potential $[\mathrm{EZA}],[\mathrm{JPH}]$.

It is worth pointing out that the field-theoretical generalization of the Girvin model is the non-relativistic Maxwell-Chern-Simons system proposed by Manton [MAN], whose 2+1-dimensional Lagrangian reads

$$
\frac{1}{2} b^{2}-\frac{i}{2}\left(\psi^{*} D_{t} \psi-\psi\left(D_{t} \psi\right)^{*}\right)+|\vec{D} \psi|^{2}+U(\psi)-\frac{\kappa}{2}\left(b a_{t}+\vec{a} \times \vec{e}\right)+a_{t}+\vec{a} \cdot \vec{\jmath}^{T},
$$

where the constant vector $\vec{j}^{T}$ (called the transport current) has been included for the sake of Galilean invariance. Note also the absence of an electric term. In a suitable Galilei frame $\vec{\jmath}^{T}$ vanishes [MAN] and, for $U(\psi) \equiv 0$ and identifying $a_{t}$ with $-i \phi$, the modified Girvin system (1) is recovered, when time-independence is assumed.

So far, we have been working with a spinless matter field. Introducing spin would not change the situation, though. Modifying the Lagrangian (1) as

$$
\mathcal{L}_{\text {spin }}=\frac{1}{2} b^{2}+(\vec{D} \Psi)^{\dagger}(\vec{D} \Psi)+i \phi\left(\Psi^{\dagger} \Psi-1\right)-b \Psi^{\dagger} \sigma_{3} \Psi-i \frac{\kappa}{2}(b \phi+\vec{a} \times \vec{\nabla} \phi)
$$

where $\Psi$ is a 2 -component Pauli spinor, would replace (2a) by the Pauli equation

$$
i \phi \Psi=\left[\vec{D}^{2}+b \sigma_{3}\right] \Psi
$$

while (2b) and (2c) remain unchanged up to $\vec{\jmath}=-i\left(\Psi^{\dagger} \vec{D} \Psi-(\vec{D} \Psi)^{\dagger} \Psi\right)+\vec{\nabla} \times\left(\Psi^{\dagger} \sigma_{3} \Psi\right)$. Then, for $\kappa= \pm(1 / 2)$, the spinning system admits self-dual solutions of definite chirality, $\Psi_{+}=\left(\begin{array}{c}0 \\ \psi_{+}\end{array}\right)$and $\Psi_{-}=\left(\begin{array}{c}\psi_{-} \\ 0\end{array}\right)$, with $\varrho=\left|\Psi_{ \pm}\right|$satisfying the same Liouville-type equation (5) [HHY].

In Ref. $[\mathrm{ZH}]$, Zhang et al. proposed another 'Landau-Ginzburg' theory for the QHE. They consider a scalar field $\psi$ coupled to a gauge field $A_{\mu}$, described by the Lagrangian

$$
\begin{aligned}
\mathcal{L}_{Z}= & 4 \theta \epsilon^{i j}\left(2 A_{0} \partial_{i} A_{j}-A_{i} \partial_{0} A_{j}\right)-\frac{1}{4 \theta} \epsilon^{\mu \nu \sigma} A_{\mu} \partial_{\nu} A_{\sigma} \\
& +\psi^{*}\left[i \partial_{0}-\left(A_{0}+A_{0}^{e x t}\right)\right] \psi+\psi^{*}\left[-i \vec{\nabla}-\left(\vec{A}+\vec{A}^{e x t}\right)\right]^{2} \psi+U(\psi) .
\end{aligned}
$$

where $A_{\mu}^{e x t}$ is the vector potential of an external electromagnetic field, and $U(\psi)=\mu|\psi|^{2}-\lambda|\psi|^{4}$ is a quartic self-interaction potential. They argue that their theory is different from that of Girvin and MacDonald. Now we show that for a static system in a purely magnetic background and for $U(\psi) \equiv 0$, the two models are indeed mathematically equivalent. To see this, let us note that under the above restrictions, after some partial integrations and dropping surface terms, the Lagrangian of Zhang et al. becomes

$$
\left(4 \theta-\frac{1}{4 \theta}\right) \epsilon^{\mu \nu \sigma} A_{\mu} \partial_{\nu} A_{\sigma}-A_{0}|\psi|^{2}+\mid\left(-i \vec{\nabla}-\left.\left(\vec{A}+\vec{A}^{e x t}\right) \psi\right|^{2} .\right.
$$


On the other hand, the Girvin-MacDonald model can also be presented in a slightly different way. Let us indeed consider a static, purely magnetic external field $B^{e x t}$. Then, setting $\vec{a}=-\vec{A}-\vec{A}^{e x t}$ and $A_{0}=-i \phi$, we find that, for the choice

$$
\kappa=\frac{1}{B^{e x t}}
$$

the (original) Girvin-MacDonald Lagrangian [i.e., (1) without the $b^{2} / 2$ term] becomes, up to a surface term,

$$
\mathcal{L}_{G}=\left|\vec{\nabla}-i\left(\vec{A}+\vec{A}^{e x t}\right) \psi\right|^{2}-A_{0}|\psi|^{2}-\frac{\kappa}{2}\left(A_{0} \vec{\nabla} \times \vec{A}+\vec{A} \times \vec{\nabla} A_{0}\right)
$$

which is indeed (18) when $\kappa=-8 \theta+1 / 2 \theta\left({ }^{1}\right)$.

In Ref. [EZA] Ezawa et al. have shown that the model of Zhang et al. admits, for a suitable choice of the self-interaction potential $U(\psi)$, topological as well as non-topological vortex solutions. In the light of our results we see that, alternatively, we can add a $b^{2}$ term to the Lagrangian while still working with $U(\psi)=0$.

As to the physical significance of our solutions, our "topological" vortices correspond to quasiparticles and quasiholes. The physical interpretation of our "non-topological" vortices is, however, not yet clear. We are currently working on this issue.

Acknowledgements. M. H. and J.-C. Y. acknowledge the Laboratoire de Mathémathiques et de Physique Théorique of Tours University for hospitality. They are also indebted to the French Government and the Gouvernement de La Côte d'Ivoire respectively, for doctoral scholarships. P. A. H. would like to thank Drs. G. Dunne and R. Jackiw for discussions and the hospitality in the Center for Theoretical Physics at MIT, where part of this work was completed.

\section{References}

[GIR] S. M. Girvin, in The Quantum Hall Effect, edited by R. E. Prange and S. M. Girvin (Springer Verlag, N. Y. 1986), Chapt. 10. See also S. M. Girvin and A.-H. MacDonald, Phys. Rev. Lett. 58, 303 (1987).

[LP] E. M. Lifshitz and L. P. Pitaevski, Statistical Physics, Part 2 (Landau-Lifshitz Course of Theoretical Physics Vol. 9), Butterworth-Heinemann: Oxford (1995).

[MAN] N. Manton, Ann. Phys. (N.Y.) 256, 114 (1997). Related results can also be found in I. Barashenkov and A. Harin, Phys. Rev. Lett. 72, 1575 (1994); Phys. Rev. D52, 2471 (1995); P. Donatis and R. Iengo, Phys. Lett. B320, 64 (1994); K. Lee and P. Yi, Phys. Rev. D52, 2412 (1995).

[JP] R. Jackiw and S-Y. Pi, Prog. Theor. Phys. Suppl. 107, 1 (1992); G. Dunne, Self-Dual Chern-Simons Theories. Springer Lecture Notes in Physics. New Series: Monograph 36. (1995).

$\left(^{1}\right)$ In the reprint volume of M. Stone: Quantum Hall Effect, (Singapore: World Scientific, 1992), the first term in Eq. (17) has been suppressed. This does not change the conclusion : the two models are still equivalent when $\kappa=1 / 2 \theta$. 
[HHY] M. Hassaïne, P. A. Horváthy and J.-C. Yera, Non-relativistic Maxwell-Chern-Simons vortices, Annals of Physics (N. Y.). 263, 276 (1998).

[BOG] E. B. Bogomolny, Sov. J. Nucl. Phys. 24, 449 (1976); H. J. De Vega and F. A. Schaposnik, Phys. Rev. D14, 1100 (1976).

[ZH] S. C. Zhang, T. H. Hansson, S. Kivelson, Phys. Rev. Lett. 62, 307 (1989).

[EZA] Z. F. Ezawa, M. Hotta and A. Iwazaki, Phys. Rev. D44, 452 (1991).

[JLW] R. Jackiw, K. Lee and E. Weinberg, Phys. Rev. D42, 3488 (1990).

[WEIN] E. Weinberg, Phys. Rev. D19, 3008 (1979); C. H. Taubes, Commun. Math. Phys. 72, 277 (1980).

[JPH] R. Jackiw and S-Y. Pi, Phys. Rev. Lett. 67, 415 (1991); Phys. Rev. D44, 2524 (1991); M. Hotta Prog. Theor. Phys. 86, 1289 (1991). 